\title{
The Present Situation and Countermeasure of Compilation and Research of Archives in Colleges and Universities
}

\author{
Liu Xiqiao \\ Archives of Northeast Normal University \\ Changchun, Jilin Province, China \\ 84009476@qq.com
}

\begin{abstract}
The archives in colleges and universities are the products of the cultural accumulation of colleges and universities, the proof of the cultural progress of colleges and universities, and the inheritance of the cultural development of colleges and universities, which can't be neglected and ignored in the cultural construction of colleges and universities. After the reform and opening up to the outside world, China's colleges and universities have been developing rapidly. As an important information resource, the colleges and universities archives play an increasingly important role in the construction and development of colleges and universities. The compilation and research of colleges and universities archives takes the top priority in the whole development of archives in colleges and universities, which is directly related to the quality of archives work. At present, the development of archives work in colleges and universities is developing rapidly, but there are still many shortcomings. For instance insufficient recognition of colleges and universities, professional talent shortage, studying form is single, studying results are not good and so on. The author worked in the archives in colleges and universities for a long time, so the author has put forward some suggestions. Firstly, the author put forward the significance of studying on the archives work. Such as the important role of cultural inheritance, the function of becoming an important part of the memory of colleges and universities, and the function of providing relevant materials and technical assistance to other departments in universities and so on. Therefore, some innovative suggestions are proposed for the above problems. For instance, provide professional guidance for the research of archives work, and provide a new channel for the research work by means of the big data information, and provide special fund support for the archives work in colleges and universities. The innovation of this paper is not only to start from the theoretical level, but also to put forward the relevant methods for the university archives research work from the practice level. With the continuous rise of China's economic level and comprehensive strength, the people's pursuit of spiritual culture is getting higher and higher. Strengthening the construction of archives culture in colleges and universities can promote the healthy development of colleges and universities, cultivate better talents for the society, and provide the universities' own power for the strategy of powerful cultural country.
\end{abstract}

Keywords-colleges and universities; archives compilation and research; archives work

\section{INTRODUCTION}

Archives are the country's wealth and resources, as well as the important information reserves. Archives are of a variety of types, and in many archives centers, the colleges and universities archive is an indispensable type. The colleges and universities archives not only record the teaching and education process of colleges and universities, but also record a series of activities, such as document, accounting, school history, personnel, audio and video, and have rich instructional, educational and cultural value. While, in the process of serving the community and the school, colleges and universities archives compilation and research work emerges from an integrated file resources and development and utilization. Archives compilation and research work, which essence is the edition and research for the archives. In archives compilation and research work, the archives sectors compile relevant reference materials, compilation archives files or write papers in accordance with the archives files and actual needs on the basis of history of archives, which are the concentration and wisdom crystallization of the school teaching, science, research and management activities. In these years, colleges and universities archives compilation and research work has made some achievements, but has still faced many problems, such as insufficient professional staff, single form, low result utilization, deficient expenditure and a series of other problems For this purpose, this article will have an exploration in terms of such issues.

\section{THE SIGNIFICANCE OF ARCHIVES RESEARCH AND DEVELOPMENT WORK IN COLLEGES AND UNIVERSITIES}

\section{A. Colleges and universities archives compilation and} research work has a positive role in the cultural heritage of colleges and universities.

Colleges and universities archives compilation and research work is an important means and main force of excellent traditional culture and campus cultural inheritance. The history, the teaching experience, the accumulation of college teachers' wisdom and the development of social things are embodied in archives, which are all the precious wealth and important resources to maintain and promote the continuation and development of human civilization. As an important carrier of 
cultural heritage in colleges and universities, colleges and universities archives have an irreplaceable role in the transmission of the excellent cultural achievements created by the Chinese nation and the accumulation of knowledge and wisdom of colleges and universities scholars, moreover, they are not only the valuable resources that the excellent culture can be lasted and inherited, but also conduce to promote the construction of the socialist cultural power with Chinese characteristics.

\section{B. Colleges and universities archives compilation and} research work provide detailed and accurate information resources for the various departments of colleges and universities

Colleges and universities archives record the whole process of school development in all periods, and have detailed resource base and high scientific research value. Through the scientific analysis of the archives, Colleges and universities archives research and development work rejects the dross and assimilates the fine essence, discards the false and retains the true, and has a comprehensive processing, then the accurate and reliable archives information is divided into classes and divisions, and the archives series compilation is formed, which greatly facilitates the consultation and utilization for the various departments of the school, school teachers and social staff, so that the transfer personnel get their the file information what they need quickly, easily and accurately.

\section{Colleges and universities archives compilation and research work makes a positive contribution to the cause of colleges and universities development}

In accordance with the actual development of the school and the actual needs of various departments in the work, university archives departments carry through archival compilation and research work in line with a school-based, service-oriented and innovation-oriented development concept. Scientific collecting, selecting, processing and finishing the literature, research and development competitive products are compiled with historical reference value, resource information integration, file convenience and other characteristics. The detailed and accurate information is provided for the future innovation and development of the school, brilliant show of school history, leadership decision-making, serving teachers and students.

\section{Colleges and universities archives compilation and research work makes archives work more systematic and holistic}

School archives of various departments are complex and numerous. Many of the information alone do not seem to have any problems, but once these archival materials are collected, it will be found that there are many contradictions and errors. It has been found when archives departments research and compile various types of archives that these departments are sometimes fragmented, resulting in many work to repetition, out of touch, however, archives work turns the isolated and resting archives into systematic and dynamic materials. Therefore, through compilation and research work, the deficiencies and errors in management work can be found in time.

\section{E. Colleges and universities archives compilation and research work is an important part to build the university memory.}

Colleges and universities archives compilation and research work truly records the historical development track of colleges and universities, campus celebrities, minutes and other records, vividly reappearing the whole picture of the college personnel training, scientific research and social services development. Major turning points, important events, important tasks and many other aspects of the contents during the various historic periods of colleges and universities are reflected in the files, which are an important part to build a university memory, are also a brilliant historical memory recordation.

\section{THE STATUS OF COLLEGES AND UNIVERSITIES ARCHIVES COMPILATION AND RESEARCH WORK}

\section{A. There are short talents in college archives compilation and research work, and their comprehensive abilities need to} be improved.

Personnel's good political quality and professional quality are the premise and security of a good job in college archives compilation and research work, and thus the higher requirements are put forward for the overall quality of editorial and research staff. Because of the impact of traditional ideas, many colleges and universities think that the archives work is very simple, nothing more than to collect, organize, take the use of the work, does not need professionals, only need some people to watch the archives.[1] Therefore, many colleges and universities have few full-time archives staff, many people who are holding several posts simultaneously, so it is difficult for them to put more time and effort to the file editing work. Colleges and universities archives editors have lower education, especially are lack of computer network personnel, the overall educational background level of existing archives staff in many colleges and universities academic are relatively low, with less professional origin, mostly are halfway monks. Their knowledge structure is not reasonable, and there is no full-time file editing staff, which have resulted in that there is no way to start large numbers of archives information and the file editing and research work is seriously affected due to the lack of professional knowledge in specific work. In addition, on account of the impact of their own job nature, objects and the environment, there are few foreign exchange learning opportunities for college archives staff. Therefore, their knowledge scope is difficult to expand, comprehensive management capacity and scientific research ability are relatively weak, and the overall level of editing is not high.

\section{B. Colleges and universities archives compilation and} research is in a single form, the richness of the collection needs to be increased.

In recent years, in the development process of archives research and development, it is not difficult to find that the current archives results of the existing compilation and research mainly reflect the contents of party and government 
management and teaching management aspects, while, it is relatively deficient for those which reflect the teaching experiment, scientific research, capital construction and the characteristics of the school's, they still remain in the preparation of school memorabilia, minutes, yearbooks and some other basic information. As the archives compilation and research staff are not well understand the scientific research, characteristic education, capital construction and other professional and technical work, they often feel far exceed their power to research and develop these archives information resources, and there is a tendency to heavy management and light technology. At the same time, due to the irrational structure of the archives, the content coverage is narrow, the types of materials are single, additionally, the archival information is relatively old, which seriously restrict the deepening of the archives research and development in colleges and universities. The reason is that I think in the daily use of service work of school archives, there are relatively large number of people who look up and use archives of the party and government management and teaching management, but there are relatively fewer people who look up and use archives of scientific research, characteristic education, capital construction and other files.[2] Therefore, the archives with less utilization are put into a relatively minor position, resulting in a single form of college archives and complexion of not abundant collection.

\section{The value of colleges and universities archives compilation and research is lower, and the recognition rate of scientific research needs to be strengthened}

At present, there are many colleges and universities archives compilation and research work is still in the initial stage, and archives compilation and research work is the organic combination between "compilation” and "research", so it should be done that give consideration to both compilation and research, research-from-compilation. Therefore, research effort of archives compilation and research should be increased, on the basis of the archives compilation, and the weight of the file research should be gradually increased, continuously striving for the direction of the research. As the sizes, levels and various conditions of colleges and universities archives are less than the national archives, colleges and universities archives are still taking compilation as the principle thing, lack of research. From the existing compilation and research results, most of the archives just copy and excerpt the basic data and the ready-made materials, such as memorabilia, yearbooks, document compilations, organizational evolution, conference briefings and other basic research topics, neglecting the aspect of on the basis of compilation. This behavior of just compilation not research and simple summary eventually led to the low value of archives compilation and research results and low utilization rate.

\section{THE STRATEGIES OF ARCHIVES COMPILATION AND RESEARCH WORK IN COLLEGES AND UNIVERSITIES}

Archives compilation and research work is the important means of archives information utilization service and an important part of file management. At present, with the deepening of China's reform and opening up, the cause of higher education is also developing rapidly. Archives work is playing an increasingly important role in the process of colleges and universities survival and development. Among them, archives compilation and research work is particularly important. The following proposes a constructive strategy for the above issues.

\section{A. Improving the overall quality of archives staff, adding new vitality for the colleges and universities archives compilation and research work}

With the rapid development of China's society, archives compilation and research work is also constantly evolving. Archives compilation and research work has been developed from simple compilation to more complex modern management, and archivists are not past custodians. This requires archivists to change their traditional ideas and constantly improve their political quality, theoretical accomplishment and cultural literacy, even more to have over hardening professional knowledge and the ability of linking theory with practice, or otherwise, it will be difficult for them to do the job.[3] Therefore, it is necessary to improve the overall quality of the staff of the archives. Firstly, the computer network expertise of the staff should be continuously improved, it is necessary to equip with high-quality computer network technology professionals to support archives compilation and research work. Secondly, the professional moral quality of the staff of archives compilation and research work should be improved. Archives staff should love and respect their jobs, stand on solid ground, bury in the research work, ardently love archives work, processing with dedication and innovative spirit, to make extraordinary results in their ordinary posts. Thirdly, on-the-job learning and training of the staff should be strengthened, and to create a mutual learning, communication, support and proactive atmosphere in the daily work environment, continuously improving the business level of the staff. Fourthly, a good coordination and the interpersonal relationship should be created. This will help to compile materials and collect demand information.

\section{B. Enriching the resources of colleges and universities archives compilation and research materials, broadening the channels for colleges and universities archives compilation and research work}

Colleges and universities archives are the basis and source of compilation and research work, the compilation and research work can't be carried out if there is no rich collection available to be used by editors. Therefore, it is necessary to improve the qualities, broaden the channels, and enlarge the source of basic elements of compilation and research work. Specifically, this requires that, in the colleges and universities archives compilation and research work, the editorial staff should strengthen the collection and collation of archival source, and actively promote the archives work, select topics around the centers of various periods of school to carry out compilation and research work. Archivists of colleges and universities should do regular research and collection of materials, search for information resources, and urge the secondary colleges and departments to organize the information and timely transfer of archives, and important archives information should be 
transferred at any time. At the same time, as for the major projects, grave celebrations, important meetings and significant people to visit, lectures and inspections of the school, the archives departments should timely track to ensure the integrity, accuracy and timeliness of archival information, to lay a solid foundation to promote the smooth development of archives compilation and research work and to promote the overall level of archives management work in colleges and universities.[4]

\section{Improving the quality of colleges and universities archives compilation and research results, firming a solid foundation for the colleges and universities archives compilation and research work}

At present, the ultimate goal for colleges and universities' developing archives information resources and completing compilation and research work is to provide high quality and efficient services for various jobs of the school. Therefore, the topics of archives compilation and research should be closely related to the teaching, research and management in the school, so that it has the guiding role and reference value in practical work, including the following aspects: First, it will stand out the characteristics to improve the quality of archives, for example, carrying out the characteristic compilation and research to form the series of compilation and research results with characteristics of the school by virtue of the project evaluations, key laboratory constructions, famous majors and doctoral and master pilots declaration and other jobs. Second, file-oriented selection should be needed. The use of demand is the starting point of archives work. Where there is no need, there is no necessity for compilation and research. Third, a variety of methods and means should be used to carry out diversified carriers of compilation and research work, such as photography archives, video archives, photo archives and teaching and researches, teaching practices, teaching techniques, management techniques and other topics of file archives, so as to maximize excavate the value of archives resource information, improve the level and quality of compilation and research work, advance the efficiency of archives, directly provide comprehensive information for colleges and universities to strengthen management and develop scientifically, and make a positive contribution to the development of colleges and universities.

\section{Enhancing the informationization of colleges and universities archives compilation and research, providing a new channel for colleges and universities archives compilation and research}

With the advent of the digital age, there are many new forms of archives compilation and research in colleges and universities. Through the digital processing of archival information, it eliminates the drawbacks of traditional manual compilation and research, improves the efficiency of colleges and universities archives compilation and research, and effectively reduces the strength of the work of the editors of colleges and universities. However, the emergence of digital technology also puts forward higher requirements for the researchers, it is not only to grasp the latest achievements and the latest research trends of professional development of relevant disciplines, establish the connection among library, research and academic departments and other institutions, interflow the information, interchange research and research topics, but also to establish archives website, form the research and research results integrating text, sound, images, pictures, music and other forms, publish the latest research and development results online, make acoustic image archives, expand the propaganda, and promote inter-school digital file information

\section{CONCLUSION}

With the continuous improvement of China's economic level and comprehensive strength, people's requirements for spiritual culture are getting higher and higher. Nowadays, colleges and universities are an important base for cultivating talents in our country. Therefore, the healthy development of colleges and universities is taking very significant role for our country's social development. The management of archives is an important content of the managements of colleges and universities. It is only from the school itself to enhance the professionalism of the managers, to strengthen the infrastructure of the archives, to realize the rationalization of the structure of the collection function, to innovative file service, to keep up with era trend, to master the information technology, can be able to achieve the optimization of archives management. If the colleges and universities archives compilation and research work ability and archives culture construction can be strengthened, it is possible to promote the positive and healthy development of colleges and universities so as to cultivate more and better talents to serve the society.

\section{REFERENCES}

[1] Shunhong, Zhu. The Current Situation and Development Trend of Archives Work in Colleges and Universities [J]. Journal of Shaoguan College, 2006, 04 (4): 139.

[2] Xiuying, Zhou. On the Innovation of Archives Compilation and Research Work in Colleges and Universities in the New Period [J]. Zhejiang Archives, 2005 (6): 37 -38.

[3] Jin, Yan. On the Characteristics of Archives Compilation and Research Work in Network Environment of Information Era [J]. Journal of Guangdong University of Technology: Social Science Edition, 2005 (4): $76-79$.

[4] Dongmei, Zhang. Discussing Archives Compilation and Research Work in Colleges and Universities in the New Period [J]. Journal of Hebei Radio \& TV University, 2006 (4). 